\title{
A RT-PCR method for selective amplification and phenotypic characterization of all three serotypes of Sabin-related polioviruses from viral mixtures
}

\author{
Eliane Veiga da Costa ${ }^{1}$, Renata de Mendonça Campos ${ }^{1}$, Fernando Neto Tavares ${ }^{1}$, \\ Cátia Regina Valério Grégio', Fernanda Marcicano Burlandy', Edson Elias da Silva ${ }^{1}{ }^{+}$ \\ ${ }^{1}$ Laboratório de Enterovírus, Instituto Oswaldo Cruz-Fiocruz, Rio de Janeiro, RJ, Brasil
}

\begin{abstract}
Outbreaks caused by vaccine-derived polioviruses are challenging the final eradication of paralytic poliomyelitis. Therefore, the surveillance of the acute flaccid paralysis cases based on poliovirus isolation and characterization remains an essential activity. Due to the use of trivalent oral poliovirus vaccine (OPV), mixtures containing more than one serotype of Sabin-related polioviruses are frequently isolated from clinical samples. Because each poliovirus isolate needs to be individually analyzed, we designed polymerase chain reaction primers that can selectively distinguish and amplify a genomic segment of the three Sabin-related poliovirus serotypes present in mixtures, thus, optimizing the diagnosis and providing prompt information to support epidemiologic actions.
\end{abstract}

Key words: diagnostic techniques and procedures - VP1 protein - poliovirus

The control of polio in endemic regions is heavily dependent on the use of the attenuated oral poliovirus vaccine (OPV) as monovalent, bivalent or trivalent formulations (Olivé et al. 1997, Aylward \& Maher 2006). After oral application, the attenuated polioviruses (Sabin strains) replicate in the gastrointestinal tract, where mutations and recombination can occur. As a result, subpopulations containing viral phenotypic changes can emerge, which may include features of neurovirulence similar to those presented by wild polioviruses $(\mathrm{Na}-$ thanson \& Kew 2010). Samples that show more than 1\% nucleotide differences, as compared to the corresponding Sabin strain, are called vaccine-derived poliovirus (VDPV). Several poliomyelitis outbreaks associated with VDPVs have occurred in Egypt, Hispaniola (Haiti and the Dominican Republic), the Philippines, Madagascar and other countries (Minor 2009). For this reason, efforts to maintain an efficient surveillance system are necessary. The World Health Organization (WHO) gold standard for poliovirus diagnostic is based on virus isolation in cell culture followed by typing of the isolated virus and intratypic differentiation (ITD) to distinguish Sabin-related from wild polioviruses (WHO 2004) while VP1 nucleotide sequence is routinely used for VDPV screening. For single isolates (wild or Sabin-related) the primer set Q8-Y7 is generally used for VP1 sequence (Rico-Hesse et al. 1987). During laboratory procedures, it is relatively common to have more than one serotype

Financial support: IOC/FIOCRUZ, CNPq

EVC and RMC contributed equally to this work.

+ Corresponding author: edson@ioc.fiocruz.br

Received 26 October 2011

Accepted 21 March 2012 of poliovirus isolated from a given clinical sample. As each poliovirus in the mixture needs to be analyzed, it is necessary to individualize them (WHO 2004). The recommended method is the neutralization test, which is laborious, time-consuming and can delay the identification of the poliovirus. The possibility of amplifying the genomic sequence of the VP1 gene without the individualization of the virus is of great relevance to the acute flaccid paralysis surveillance. This study describes the design of primers to be used in polymerase chain reaction (RT-PCR) reactions that can selectively amplify the genomes of the three Sabin-related poliovirus serotypes in mixtures, allowing the analysis of the VP1 gene. Three forward primers corresponding to each serotype of Sabin virus (Toyoda et al. 1984) and targeting the 3'- end of the VP3 gene were designed and used in combination with a previously described reverse primer that targets the 5'- portion of the 2A gene, named Q8 (Rico-Hesse et al. 1987). A preliminary report dealing with this subject has been presented during the XXI National Meeting in Virology, 2010 (Campos et al. 2010).

Samples containing poliovirus mixtures isolated in our laboratory were used. RNA extraction and cDNA synthesis were carried out using the methods proposed by the WHO (2004). The PCR was conducted in separate reactions, according to the serotypes, and is briefly described below. The PCR mixture for each tube consisted of the following components: $3 \mu \mathrm{L}$ of cDNA, sense primer (50 pmol), antisense primer (50 pmol) (Table I), $25 \mu \mathrm{l}$ of GoTaq $^{\circledR}$ Green Master Mix (2X) (Promega) and $20 \mu \mathrm{L}$ of $\mathrm{DNase} / \mathrm{RNase}$-free water. The reaction parameters were 25 cycles, consisting of $95^{\circ} \mathrm{C}$ for $30 \mathrm{~s}, 55^{\circ} \mathrm{C}$ for $30 \mathrm{~s}$ and $72^{\circ} \mathrm{C}$ for $2 \mathrm{~min}$, with a final extension at $72^{\circ} \mathrm{C}$ for $7 \mathrm{~min}$. The specific products were gel purified using QIAquick Gel Extraction Kit (Qiagen). Cycle sequencing reactions were performed using the ABI Prism BigDye Terminator v.3.1 kit (PE Applied-Biosystems, Foster City, CA, USA) with 25 cycles at $96^{\circ} \mathrm{C}$ for $25 \mathrm{~s}, 50^{\circ} \mathrm{C}$ for $25 \mathrm{~s}$ and 
$60^{\circ} \mathrm{C}$ for $3 \mathrm{~min}$, and the samples were analyzed using the ABI PRISM 310 Genetic Analyzer (Applied Biosystems, Foster City, CA, USA). To confirm the specificity of the primers, cDNA from each Sabin prototype strain was prepared and tested by amplification with the three primer pairs (S1-Q8, S2-Q8 and S3-Q8). No nonspecific reaction among the Sabin serotypes was observed in our analyses (Fig. 1A). Also, the specificity of the primer pairs was tested across the reactions using wild poliovirus types 1 and 3, enteroviruses from species $\mathrm{C}$ and poliovirus mixtures that were previously isolated (II). The primer pairs were unable to amplify the wild polioviruses or the species $C$ enterovirus; however, recently isolated type 2 Sabin-related strains, presenting four and

TABLE I

Nucleotide sequence of the primers used in the polymerase chain reaction

\begin{tabular}{lcc}
\hline Primer & $\begin{array}{c}\text { Sequence } \\
\left(5^{\prime}-3^{\prime}\right)\end{array}$ & $\begin{array}{c}\text { Position } \\
(\mathrm{nt})^{c}\end{array}$ \\
\hline $\mathrm{Q}^{a}$ & 5'-ACYAAGAGGTCTCTATTCCAC-3' & $3487-3507$ \\
$\mathrm{~S}^{b}$ & 5'-CAGAATAGTCGTCCCTCTTTCG-3' & $2361-2382$ \\
$\mathrm{~S}^{b}$ & 5'-TAGGGTTGTTGTCCCGTTGTCC-3' & $2361-2382$ \\
$\mathrm{~S}^{b}$ & 5'-GTCCACCCCTAAGAGTATGAG-3' & $2379-2401$ \\
\hline
\end{tabular}

$a$ : anti-sense primer; $b$ : sense primer; $c$ : position relative to the positions reported by Toyoda et al. (1984). six mutations, respectively, in the VP1 region, were correctly amplified. The same was true for all of the poliovirus mixtures detected at the enterovirus laboratory during 2010 (Fig. 1B, Table II) (Campos et al. 2010). The nucleotide sequences obtained were deposited in GenBank (JN688967-JN688978). These results proved the utility of the primer pairs described herein, especially in field samples where the amount of particles can vary due to the competition between serotypes during repli-

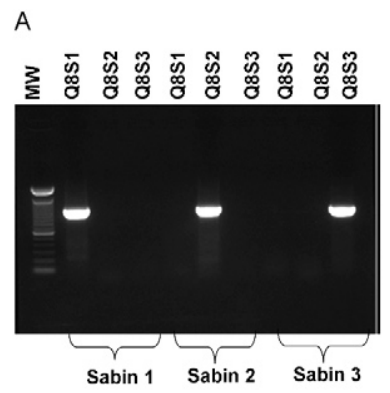

B

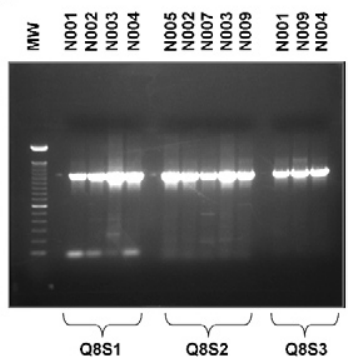

Fig. 1: agarose $1 \%$ gel electrophoresis showing amplicons generated by each of the Sabin serotype specific primers used in combination with the primer Q8. A: Lanes 1-3: reference Sabin 1 tested with primer pairs Q8-S1, Q8-S2 and Q8-S3, respectively; 4-6: reference Sabin 2 tested with primer pairs Q8-S1, Q8-S2 and Q8-S3, respectively; 7-9: reference Sabin 3 tested with pairs Q8-S1, Q8-S2 and Q8-S3; B: Lanes 2-5: samples N001, N002, N003 and N004 amplified with primer pair Q8-S1; 7-11: samples N005, N002, N007, N003 and N009 amplified with primer pair Q8-S2; 13-15: samples N001, N009 and N004 amplified with primer pair Q8-S3; MW: molecular weigh.

\section{TABLE II}

Sample information and results of the described polymerase chain reaction (RT-PCR)

\begin{tabular}{|c|c|c|c|c|}
\hline Samples & $\begin{array}{c}\text { Year } \\
\text { of isolation }\end{array}$ & Serotype & $\begin{array}{c}\text { RT-PCR } \\
\text { tested/positive } \\
(\mathrm{n} / \mathrm{n})\end{array}$ & $\begin{array}{l}\text { GenBank } \\
\text { access }\end{array}$ \\
\hline \multirow[t]{3}{*}{$\begin{array}{l}\text { Vaccine-related } \\
\text { poliovirus }\end{array}$} & 2010 & $\begin{array}{l}\text { Mixture } \\
(\mathrm{P} 1+\mathrm{P} 2)\end{array}$ & $2 / 2$ & $\begin{array}{l}\text { (P1N002)-JN688968 } \\
\text { (P2N002)-JN688972 } \\
\text { (P1N003)-JN688969 } \\
\text { (P2N003)-JN688974 }\end{array}$ \\
\hline & 2010 & $\begin{array}{l}\text { Mixture } \\
(\mathrm{P} 1+\mathrm{P} 3)\end{array}$ & $2 / 2$ & $\begin{array}{l}\text { (P1N001)-JN688967 } \\
\text { (P3N001)-JN688976 } \\
\text { (P1N004)-JN688970 } \\
\text { (P3N004)-JN688978 }\end{array}$ \\
\hline & 2010 & $\begin{array}{l}\text { Mixture } \\
(\mathrm{P} 2+\mathrm{P} 3)\end{array}$ & $1 / 1$ & $\begin{array}{l}\text { (P2N009)-JN688975 } \\
\text { (P3N009)-JN688977 }\end{array}$ \\
\hline $\begin{array}{l}\text { Vaccine-derived } \\
\text { poliovirus and pre-derived }\end{array}$ & 2010 & $\mathrm{P} 2$ & $2 / 2$ & $\begin{array}{l}\text { (P2N005)-JN688971 } \\
\text { (P2N007)-JN688973 }\end{array}$ \\
\hline Wild poliovirus & $\begin{array}{c}1988 / 1981 \\
1988\end{array}$ & $\begin{array}{l}\text { P1 } \\
\text { P3 }\end{array}$ & $\begin{array}{l}4 / 0 \\
4 / 0\end{array}$ & - \\
\hline Specie C enterovirus & $2006 / 2009$ & CA13, CA24, EV99 & $3 / 0$ & - \\
\hline
\end{tabular}

CA: coxsackievirus; EV: enterovirus; P: poliovirus. 


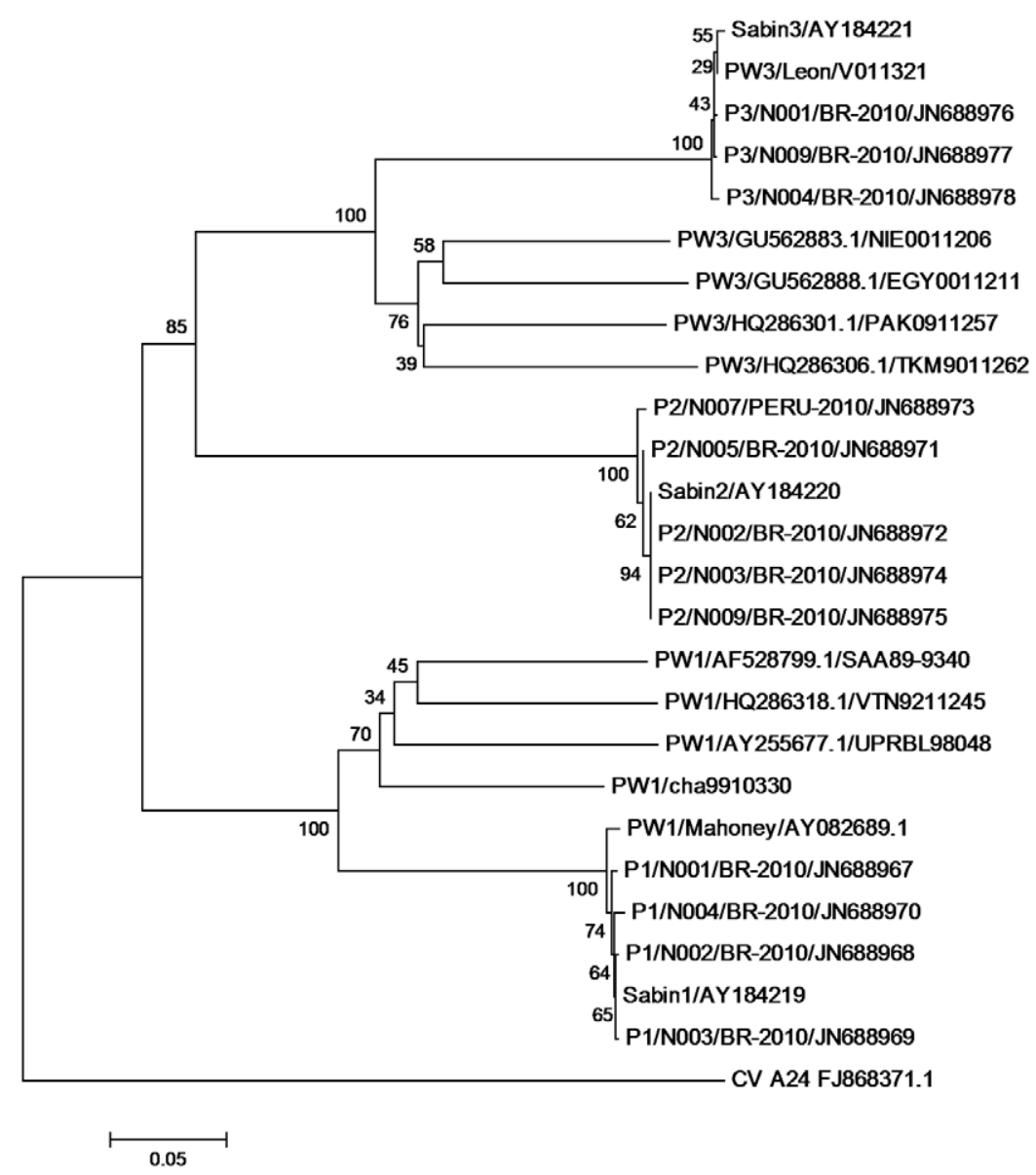

Fig. 2: dendrogram based on complete VP1 sequence was constructed by the neighbour joining method through Kimura-two parameter model from Molecular Evolutionary Genetics Analysis software version 4.0 with 1,000 pseudoreplicates. All sequences obtained from the polymerase chain reaction amplification, as well as sequences of wild poliovirus and a specie C enterovirus (CA24) were included.

cation in the cell culture. The primer pairs also showed good performance for sequencing the Sabin-related polioviruses (Fig. 2). In addition, Sabin isolates showing high rates of mutation in VP1 (Table II) were perfectly amplified. With respect to the inability of the proposed primers to amplify wild viruses, it is important to emphasize that the methodology proposed aims to amplify mixtures of Sabin serotypes and not mixtures of Sabin and wild viruses. This method should be used as a final step of further characterization of vaccine-related poliovirus mixtures isolated and identified according to the flowchart of tests recommended by WHO (2004).

The detection of wild poliovirus is achieved in the ITD step as mentioned before. The methodology for sequence single isolates of wild virus or potential VDPV is worldwide known. Recently, a first report describing the potential usefulness of a group of primers for further characterization of wild poliovirus in mixtures was described by Kilpatrick et al. (2011). Those primers were designed mainly to focus on endemic areas where wild poliovirus is still circulating. The RT-PCR proposed herein proved to facilitate the diagnostic routine of nonendemic areas where OPV is still in use. Considering its simplicity and efficiency, we believe that it can be widely used by other laboratories involved in poliovirus diagnosis and research groups. Under the new scenario, where an outbreak associated with VDPV is an emergent situation, the adoption of the RT-PCR described herein can optimize the routine testing of reference laboratories, thereby reducing the time of the analyses and providing accurate and prompt information to support epidemiological actions.

\section{REFERENCES}

Aylward RB, Maher C 2006. Interrupting poliovirus transmission new solutions to an old problem. Biologicals 34: 133-139.

Campos RM, Tavares FN, da Silva EE, da Costa EV 2010. A RT-PCR method for selective amplification and phenotypic characterization of all three serotypes of Sabin-related polioviruses from viral mixtures. Abstracts of the XIX National Meeting of Virology. Virus Rev Res 15: 256-257.

Kilpatrick DR, Iber JC, Chen Q, Ching K, Yang S, De L, Mandelbaum MD, Emery B, Campagnoli R, Burns CC, Knew O 2011. Poliovirus serotype-specific VP1 sequencing primers. J Virol Meth 174: 128-130. 
Minor P 2009. Vaccine-derived poliovirus (VDPV): impact on poliomyelitis eradication. Vaccine 27: 2649-2652.

Nathanson N, Kew OM 2010. From emergence to eradication: the epidemiology of poliomyelitis deconstructed. Am J Epidemiol 172: 1213-1229.

Olivé JM, Risi JB, de Quadros CA 1997. National immunization days: experience in Latin America. J Infect Dis 175: 189-193.

Rico-Hesse R, Pallansch MA, Nottay BK, Knew OM 1987. Geo- graphic distribution of wild poliovirus type 1 genotypes. Virology 160: 311-322.

Toyoda H, Kohara M, Kataoka M, Suganuma Y, Omata T, Imura T, Nomoto NA 1984. Complete nucleotide sequences of all three poliovirus serotype genomes: implication for genetic relationship, gene function and antigenic determinants, J Mol Biol 174: 561-585.

WHO - World Health Organization 2004. Polio laboratory manual, 4th ed., WHO, Geneve, 101 pp. 